\title{
MicroRNA-495 regulates human gastric cancer cell apoptosis and migration through Akt and mTOR signaling
}

\author{
JUN WANG $^{1 *}$, WEIWEI FENG ${ }^{2 *}$, YUANQIANG DONG ${ }^{1}$, XIANG MAO $^{1}$, FENGHUA GUO $^{1}$ and FEN LUO ${ }^{1}$ \\ ${ }^{1}$ Huashan Hospital, Fudan University, Shanghai 200040; \\ ${ }^{2}$ The International Peace Maternity \& Child Health Hospital of China Welfare Institute, \\ Shanghai Jiao Tong University School of Medicine, Shanghai 200030, P.R. China
}

Received September 28, 2017; Accepted August 7, 2018

DOI: $10.3892 /$ or.2018.6722

\begin{abstract}
In the present study, we investigated the expression and cellular distribution of microRNA-495 (miR-495) in human gastric cancer. Prominent downregulation of miR-495 activation was evident in patients with gastric cancer. Cell viability and Annexin V/PI apoptosis assays were used to assess cell proliferation and apoptosis. Then, western blot analysis was used to assess cyclin D1, PI3K, p-Akt and p-mTOR protein expression. Overexpression of miR-495 significantly inhibited cell proliferation, and promoted cell apoptosis of gastric cancer cells. Overexpression of miR-495 also promoted caspase-3/-9 and Bax protein expression, and suppressed cyclin D1 protein expression and the PI3K/Akt/mTOR pathway in gastric cancer cells. Downregulation of miR-495 increased cell proliferation and inhibited cell apoptosis of gastric cancer cells through activation of the PI3K/Akt/mTOR pathway. The PI3K inhibitor, was used to suppress the PI3K/Akt/mTOR pathway, inhibit cell proliferation, promote cell apoptosis, promote caspase-3/-9 and Bax protein expression, and suppress cyclin D1 protein expression of gastric cancer cells through miR-495 inhibition. In conclusion, miR-495 is an important regulator of human gastric cancer cells and may contribute to cell apoptosis through the PI3K/Akt/mTOR/Bax-caspase-3/-9 and cyclin D1 pathway.
\end{abstract}

\section{Introduction}

Gastric cancer is one of the most common malignancies in China. Its morbidity is ranked second place among malignancies, respectively; while its mortality is ranked third

Correspondence to: Dr Fenghua Guo or Dr Fen Luo, Huashan Hospital, Fudan University, 12 Urumqi Road, Shanghai 200040, P.R. China

E-mail: gzxd3978473@126.com

E-mail: 13801774546@163.com

*Contributed equally

Key words: microRNA-495, human gastric cancer, autophagy, PI3K/Akt, caspase-3/-9, cyclin D1 place, as is indicated in the annual analysis of morbidity and mortality of malignancies (1). Both morbidity and mortality of gastric cancer in urban areas is ranked third place among malignancies, while these are ranked first and second place in rural areas, respectively, making gastric cancer the major cause of cancer related-deaths in rural areas in China (1).

Therefore, exploring a simple detection method with high patient compliance, excellent sensitivity and specificity for the early diagnosis is the key to enhancing the early diagnosis rate of gastric cancer and improving prognosis for patients with gastric cancer (2). It has been discovered through research in recent years that microRNA (miRNA) plays an important role in the genesis, development, invasion and metastasis of gastric cancer (3). Tumor-associated miRNAs are continuously being discovered as molecular biology continues to develop. Research on the expression of miRNA in gastric cancer, as well as its biological function and mechanism of action may provide a new direction for the diagnosis and treatment of gastric cancer (4).

miRNA is aberrantly expressed in human cancer, promotes cell transformation both in vivo and in vitro. In transgenic mice, its overexpression triggered prostate intraepithelial neoplasia (5). It was found through analyzing miRNA expression profiles of tumors with various tissue origins that miRNAs are highly expressed in multiple tumors, including head and neck squamous cell carcinoma, colon, gastric, esophageal, liver cancer and multiple myeloma (6). Furthermore, it was also ascertained in a specific confirmatory experiment regarding the expression of miRNAs in miRNAs gene are associated with increased expression to varying degrees in multiple tumor tissues, suggesting that miRNAs may be related to the genesis and development of tumors (7).

The phosphatidylinositol 3-kinase (PI3K) protein family is involved in regulating multiple cell functions, such as cell proliferation, differentiation, apoptosis and glucose transport. Increased PI3K activity has been revealed to be frequently associated with multiple cancers (8). AKT, a type of oncogene with a relative molecular weight of approximately $60 \mathrm{kDa}$, also referred to as protein kinase $\mathrm{B}$ (PKB), plays a core role in the PI3K/AKT signal transduction pathway and regulates cell proliferation, differentiation, aging, migration and apoptosis (9). mTOR is also an important kinase, and activated AKT can activate its downstream mTOR through direct and 
indirect pathways (10). mTOR mainly regulates cell autophagy through two mechanisms. At present, the extensively studied mTOR substrates are 4EBP1 and S6K, which are important regulatory factors during protein translation (11). We investigated the expression and cellular distribution of microRNA-495 (miR-495) in human gastric cancer.

\section{Materials and methods}

Cell lines and clinical samples. Human gastric cancer cell line MGC80-3 was purchased from the Cell Bank of the Chinese Academy of Sciences (Shanghai, China) and subcultured at $37^{\circ} \mathrm{C}$ in RPMI-1640 medium (Hyclone Laboratories; GE Healthcare Life Sciences, Logan, UT, USA) with $10 \%$ fetal bovine serum (FBS) and $5 \% \mathrm{CO}_{2}$. Samples from a previous study of patients with gastric cancer were included in the present study. Ethics approval of this previous study was obtained from the Ethics Committee of Huashan Hospital, Fudan University (12). Tissue samples of gastric cancer or para-carcinoma tissue were gathered and stored at $-80^{\circ} \mathrm{C}$.

Real-time PCR. Total RNA was extracted from gastric cancer or para-carcinoma tissue and $100 \mathrm{ng}$ of total RNA was reverse-transcribed into cDNA using PrimeScript ${ }^{\mathrm{TM}}$ First Strand cDNA Synthesis kit (6110A; Takara Biomedical Technology, Co., Ltd. Beijing, China). miR-495 expression was quantified using SYBR-Green Master Mix (Applied Biosystems; Thermo Fisher Scientific, Inc., Foster City, CA, USA) and a 7500 Fast Real-Time PCR system (Applied Biosystems; Thermo Fisher Scientific, Inc.) and were calculated using the $2^{-\Delta \Delta \mathrm{C}_{\mathrm{t}}}$ method.

Transduction and an inhibitor. Lentiviral vectors expressing anti-miR-495 and the negative control were purchased from Sangon Biotech Co., Ltd. (Shanghai, China). MGC80-3 cells $\left(1 \times 10^{4}\right.$ cells/well) were plated in a 6 -well plate and transduced with anti-miR-495 and the negative control for 2 days. The PI3K inhibitor (LY294002, $10 \mathrm{nM}$ ) was also added into the cells and treated for 2 days.

Cell viability assay. Following transfection, MGC80-3 cells $(2,000$ cells/well) were plated in a $96-w e l l$ plate and cultured for 1 to 3 days. For the MTT assay, MTT solution $(20 \mu \mathrm{l} ; 5 \mathrm{mg} / \mathrm{ml})$ was added into each well and incubated at $37^{\circ} \mathrm{C}$ in a humidified atmosphere with $5 \% \mathrm{CO}_{2}$ for $4 \mathrm{~h}$ and then $150 \mu \mathrm{l}$ DMSO was added into the cells for dissolution at $37^{\circ} \mathrm{C}$ for $20 \mathrm{~min}$. The absorbance values were assessed with the Epoch Microplate Spectrophotometer (BioTek Instruments, Inc., Winooski, VT, USA) at $492 \mathrm{~nm}$.

Annexin V/propidium iodide (PI) apoptosis assay. Following transfection, MGC80-3 cells ( $5 \times 10^{4}$ cells/well) were plated in a 96-well plate and cultured for 3 days. For Annexin V/PIapoptosis, the cells were washed and stained with both Annexin V and PI according to the manufacturer's instructions (BD Biosciences, Erembodegem, Belgium). Apoptosis was performed using a FACScan flow cytometer (BD FACSCalibur) and analyzed using FlowJo 7.6.1 (FlowJo LLC, Ashland, OR, USA).

Western blot analysis. The treated cells were lysed on ice using RIPA lysis buffer (Beyotime Institute of Biotechnology,
Haimen, China) for $30 \mathrm{~min}$, and then the total protein concentration was quantified utilizing a BCA protein assay kit (Beyotime Institute of Biotechnology, Haimen, China). Total protein $(30 \mu \mathrm{g})$ was separated on $8-12 \%$ SDS-PAGE gels and electrotransferred to polyvinylidene fluoride (PVDF) membranes. Then, the membranes were blocked with 5\% skim milk powder for $1 \mathrm{~h}$ and incubated with the primary antibodies: ant-LC3 (dilution 1:1,000; cat. no. ab48394; Abcam), caspase-3/-9 (dilution 1:1,000; cat. nos. sc-1224 and sc-8355; Santa Cruz Biotechnology, Inc., Santa Cruz, CA, USA), Bax (dilution 1:1,000; cat. no. sc-6236; Santa Cruz Biotechnology, Inc.), cyclin D1 (dilution 1:1,000; cat. no. sc-70899; Santa Cruz Biotechnology, Inc.), PI3K (dilution 1:1,000; cat. no sc-1332; Santa Cruz Biotechnology, Inc.), p-Akt (dilution 1:1,000; cat. no. sc-7985-R; Santa Cruz Biotechnology, Inc.), p-mTOR (dilution 1:1,000; cat. no. sc-101738; Santa Cruz Biotechnology, Inc.) and GAPDH (dilution 1:5,000; cat. no. sc-293335; Santa Cruz Biotechnology, Inc.) overnight at $4^{\circ} \mathrm{C}$, and subsequently incubated with anti-rabbit $\mathrm{IgG}$ horseradish peroxidase-conjugated secondary antibody (cat. nos. sc-2004 or sc-2005; Santa Cruz Biotechnology, Inc.), for $1 \mathrm{~h}$ at $37^{\circ} \mathrm{C}$. Proteins blank was carried out using Image_Lab_3.0 (Bio-Rad Laboratories, Inc., Hercules, CA, USA).

Statistical analysis. The results were expressed as the mean \pm SD. Significant differences between two groups were determined using one-way analysis of variance (ANOVA) followed by Tukey's post hoc test. A P-value of $<0.05$ was considered to indicate a statistically significant difference.

\section{Results}

miR-495 inactivation in patients with gastric cancer and para-carcinoma tissue, is correlated with the survival rate of gastric cancer. We identified miR-495 activation in patients with gastric cancer or para-carcinoma tissue. Gene chip and qPCR revealed that the expression level of miR-495 in patients with gastric cancer was significantly lower than that in para-carcinoma tissue (Fig. 1A and B). Next, we determined that the overall survival (OS) and disease-free survival (DFS) of the miR-495 high-expression group of patients with gastric cancer were higher than those of the miR-495 low-expression group of patients with gastric cancer (Fig. 1C and D).

Overexpression of miR-495 inhibits cell proliferation and promotes cell apoptosis of gastric cancer cells. Next, we assessed the effect of the overexpression of miR-495 (Fig. 2A) on cell proliferation and cell apoptosis of gastric cancer cells. We speculated that miR-495 may play a crucial role in MGC80-3 cells. Overexpression of miR-495 significantly inhibited cell proliferation and migration, and promoted cell apoptosis of MGC80-3 cells, compared with the negative control group (Fig. 2B-F), revealing that miR-495 was correlated with the development and progression of gastric cancer. Therefore, we next aimed to explore whether miR-495 regulated caspase-3/-9, Bax and cyclin D1 protein expression of gastric cancer cells. Western blotting results confirmed that caspase-3/-9 activity and Bax protein expression was upregulated, while cyclin D1 protein expression was suppressed in MGC80-3 cells by overexpression of miR-495, compared with the negative control group (Fig. 2G-K). 

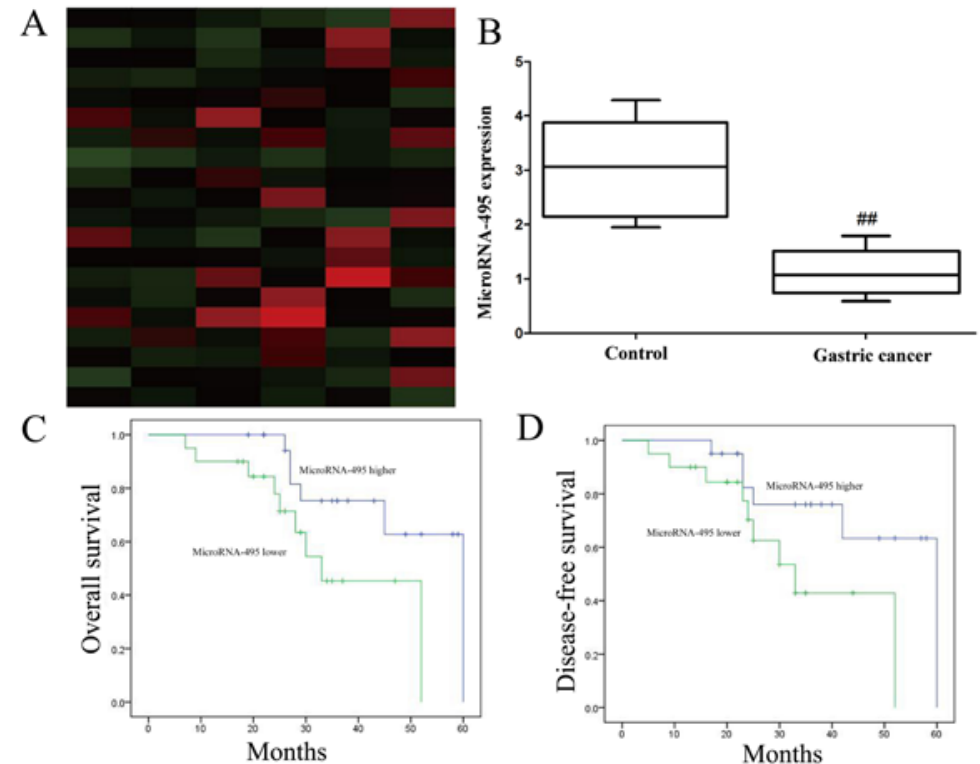

Figure 1. miR-495 inactivation in patients with gastric cancer and para-carcinoma tissue, is correlated with the survival rate of gastric cancer patients. (A) Gene chip analysis revealed the expression of miR-495. (B) qPCR revealed the expression of miR-495 in gastric cancer patients and the control. The effects of miR-495 on (C) OS and (D) DFS. ${ }^{\# \#} \mathrm{P}<0.01$ vs. the control group. miR-495, microRNA-495; OS, overall survival; DFS, disease-free survival; control, control group; gastric cancer, gastric cancer and para-carcinoma tissue.
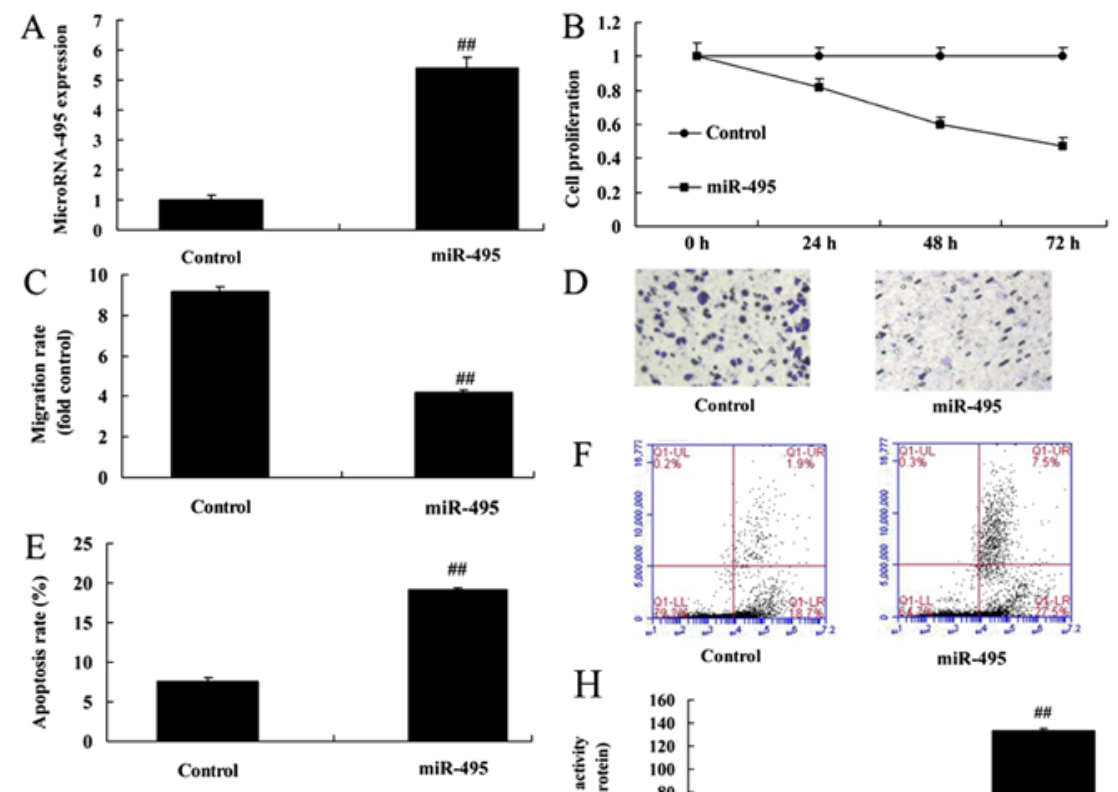

$\mathrm{D}$
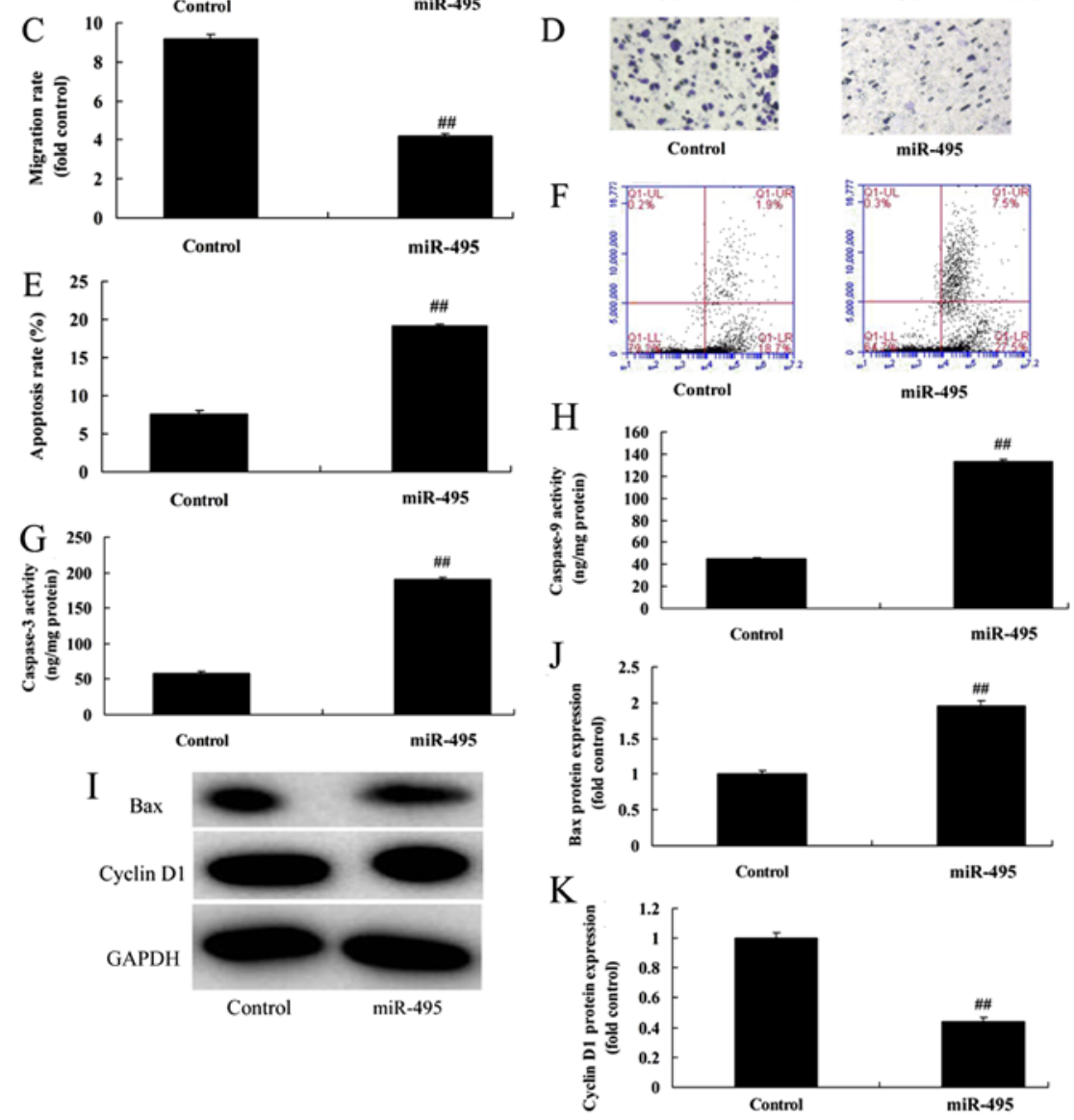

Figure 2. Overexpression of miR-495 inhibits cell proliferation and promotes cell apoptosis of gastric cancer cells. qPCR revealed (A) the expression of miR-495. (B) Cell proliferation, (C and D) migration and (E and F) cell apoptosis. (G and H) Caspase-3/-9 activity. (I-K) Bax and cyclin D1 protein expression. ${ }^{\# \#} \mathrm{P}<0.01$ vs. the control group. Control, control negative group; miR-495, overexpression of the microRNA-495 group. 

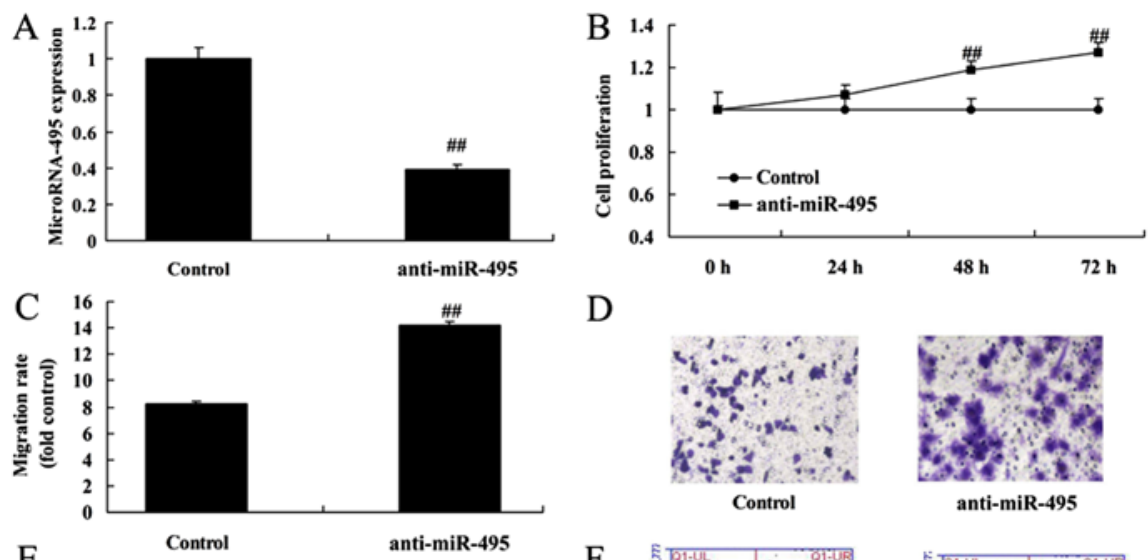

$\mathrm{D}$
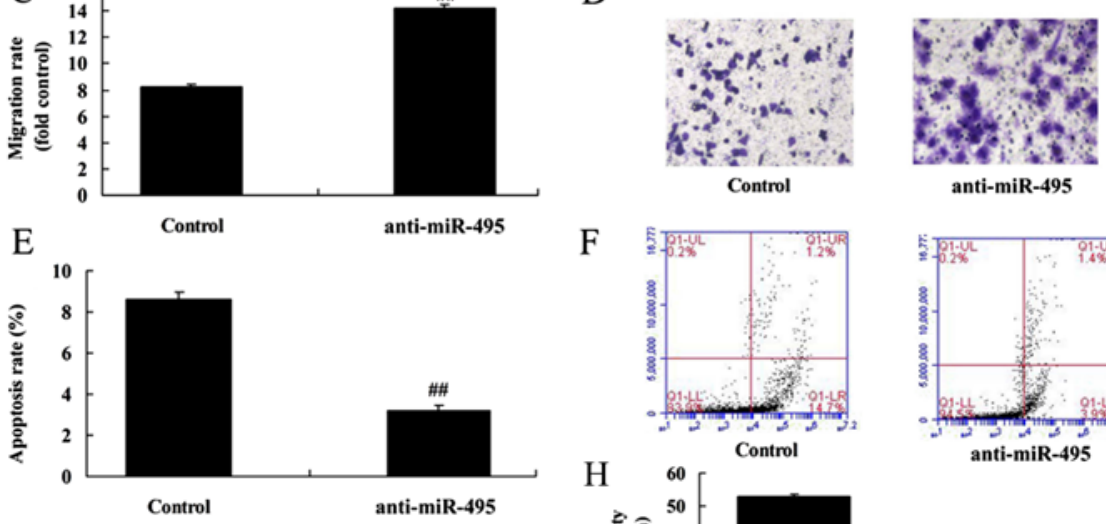

$\mathrm{F}$
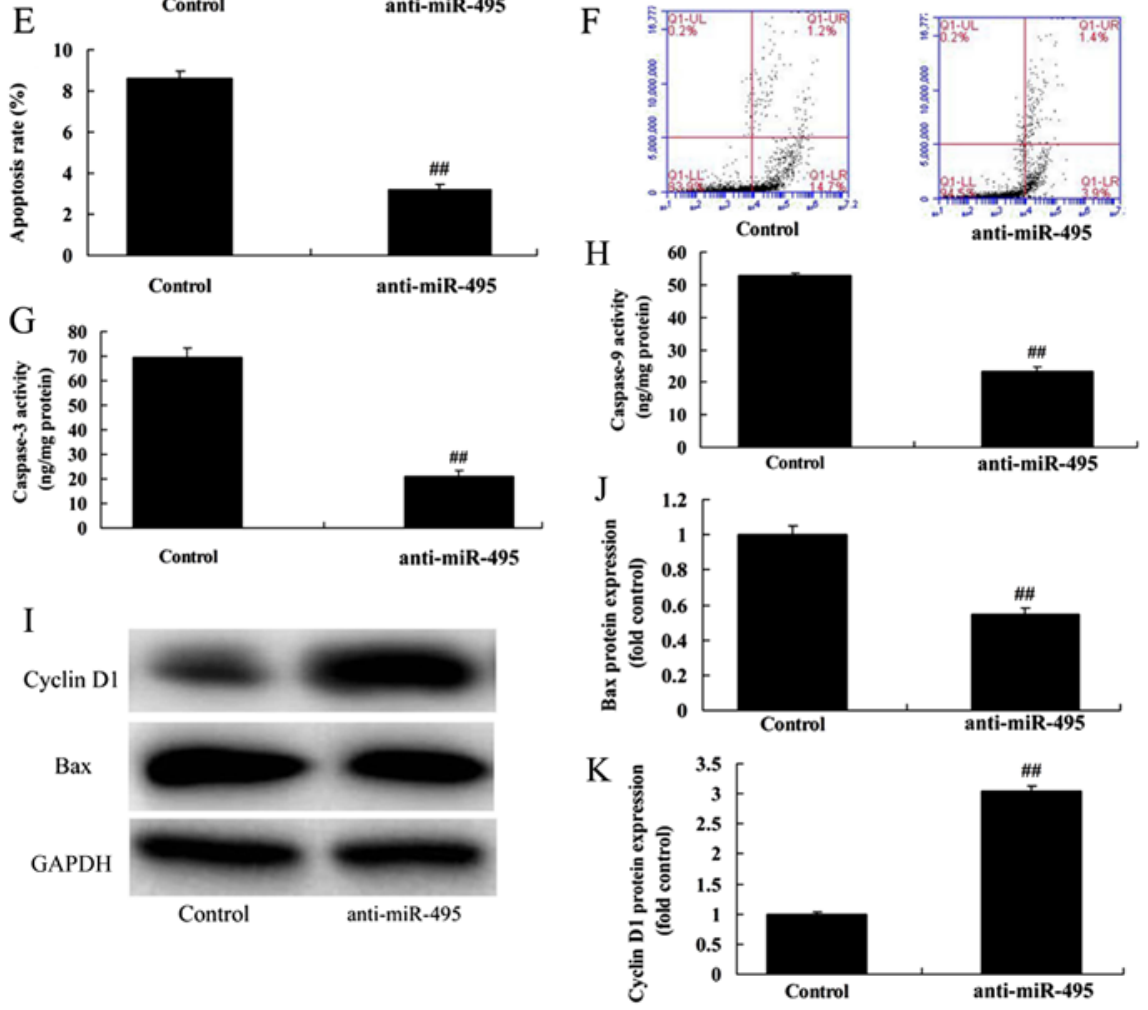

Figure 3. Downregulation of miR-495 inhibits caspase-3/-9 and Bax protein expression, and suppresses cyclin D1 protein expression of gastric cancer cells. (A) Downregulation of miR-495. (B) Cell proliferation, (C and D) migration and (E and F) cell apoptosis. (G and H) Caspase-3/-9 activity. (I) Bax and cyclin D1 protein expression using western blot analysis and ( $\mathrm{J}$ and $\mathrm{K})$ using statistical analysis. ${ }^{\# \#} \mathrm{P}<0.01$ vs. the control group. Control, control negative group; anti-miR-495, downregulation of the microRNA-495 group.

Downregulation of miR-495 increases cell proliferation and inhibits cell apoptosis of gastric cancer cells. Moreover, we then assessed the influence of anti-miR-495 on the proliferation and apoptosis of gastric cancer cells. As revealed in Fig. 3B-F, downregulation of miR-495 (Fig. 3A) significantly promoted cell proliferation and migration, and inhibited the apoptosis rate of MGC80-3 cells, compared with the negative control group. Next, we examined the impact of anti-miR-495 on caspase-3/-9, Bax and cyclin D1 protein expression of gastric cancer cells, using western blotting. The results revealed that downregulation of miR-495 significantly suppressed caspase-3/-9 activity and Bax protein expression while it induced cyclin D1 protein expression in MGC80-3 cells, compared with the negative control group (Fig. 3G-K).

miR-495 regulates the PI3K/Akt/mTOR pathway of gastric cancer cells. In addition, we applied western blotting to evaluate the PI3K/Akt/mTOR pathway in MGC80-3 cells in which miR-495 was downregulated. miR-495 had potential binding sites on the 3'-UTR of Akt and mTOR mRNAs (Fig. 4A). p-Akt and $\mathrm{p}$-mTOR protein expression was significantly suppressed by overexpression of mR-495 in MGC80-3 cells, compared with the negative control group (Fig. 4B-D). As revealed in Fig. 4E-G, downregulation of miR-495 induced p-Akt and p-mTOR protein expression in MGC80-3 cells, compared with the negative control group. The results indicated that miR-495 may affect gastric cancer cell proliferation, apoptosis and the cell cycle through the PI3K/Akt/mTOR pathway.

PI3K inhibitor suppresses the PI3K/Akt/mTOR pathway of gastric cancer cells following miR-495 downregulation. According to the aforementioned results, we used a PI3K inhibitor to suppress the PI3K/Akt/mTOR pathway of gastric cancer cells following miR-495 downregulation. LY294002 (the 


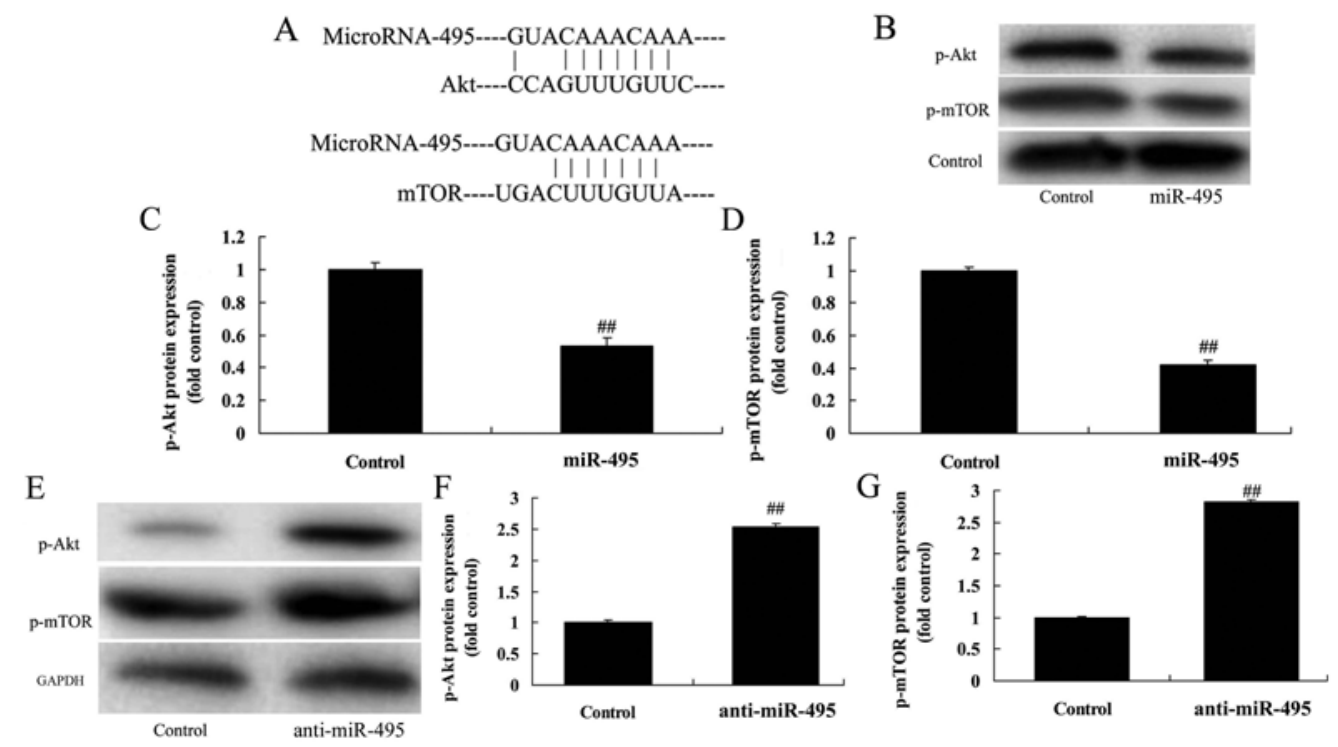

Figure 4. miR-495 regulates the Akt/mTOR pathway of gastric cancer cells. (A) miR-495 had potential binding sites on the 3'-UTR of Akt and mTOR mRNAs. (B) p-Akt and p-mTOR protein expression using western blot analysis. (C and D) p-Akt and p-mTOR protein expression using statistical analysis. p-Akt and p-mTOR protein expression following downregulation of miR-495 using $(\mathrm{E})$ western blot analysis and $(\mathrm{F}$ and $\mathrm{G})$ statistical analysis. ${ }^{\# \#} \mathrm{P}<0.01 \mathrm{vs}$. the control group. Control, control negative group; miR-495, overexpression of the microRNA-495 group; anti-miR-495, downregulation of the microRNA-495 group.
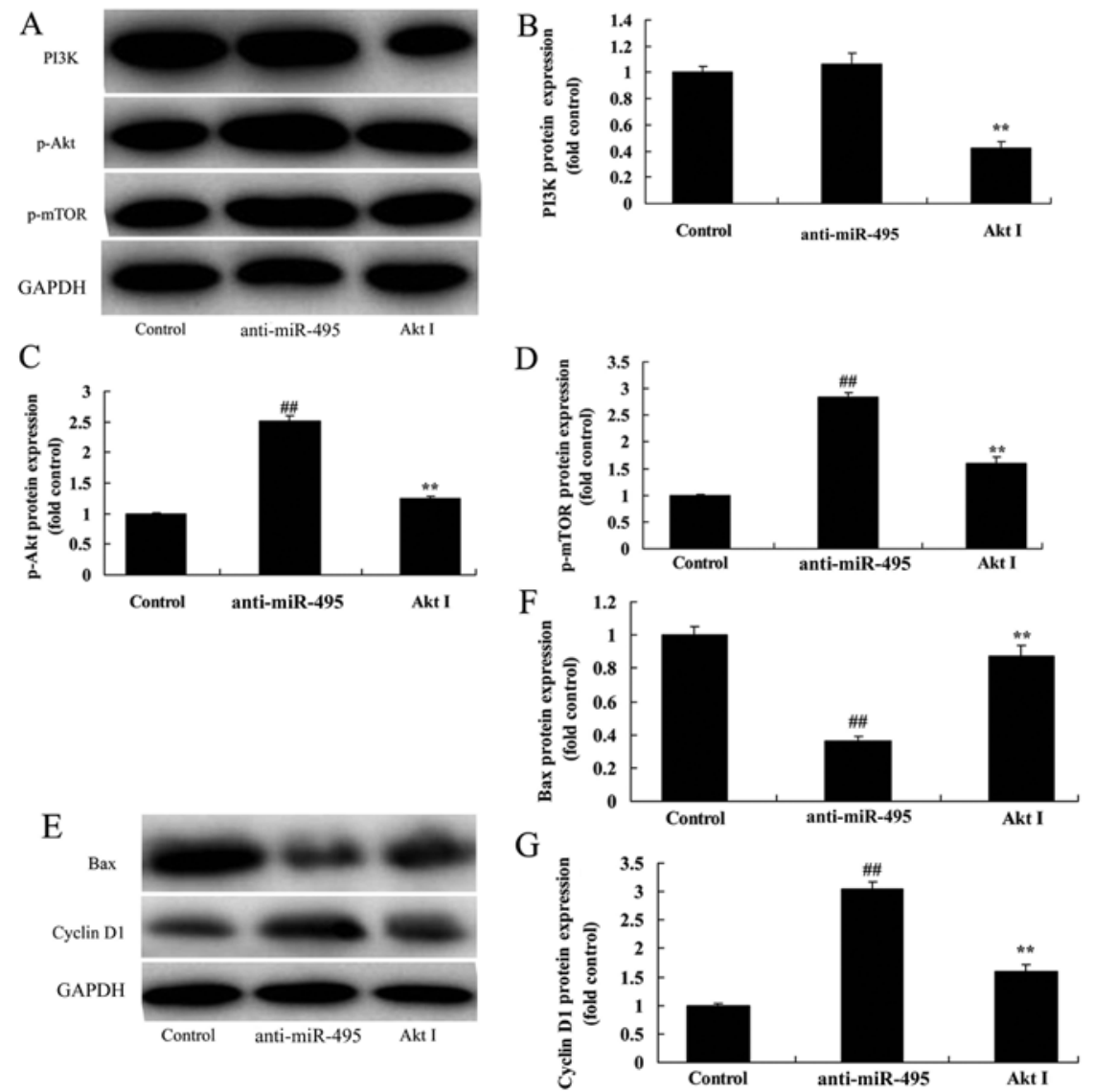

Figure 5. PI3K inhibitor suppresses the PI3K/Akt/mTOR pathway of gastric cancer cells following downregulation of miR-495. (A) PI3K, p-Akt and p-mTOR protein expression using western blot analysis and (B-D) PI3K, p-Akt and p-mTOR protein expression using statistical analysis. (E) Bax and cyclin D1 protein expression using western blot analysis and $(\mathrm{F}$ and $\mathrm{G}) \mathrm{Bax}$ and cyclin $\mathrm{D} 1$ protein expression using statistical analysis. ${ }^{\# \#} \mathrm{P}<0.01$ vs. the control group ${ }^{* * *} \mathrm{P}<0.01$ vs. the downregulated miR-495 group. Control, control negative group; anti-miR-495, downregulation of the microRNA-495 group; Akt I, downregulation of the microRNA-495 and the Akt inhibitor group.

PI3K inhibitor) could suppress the PI3K/Akt/mTOR pathway in MGC80-3 cells following miR-495 downregulation, compared with the miRNA-495 downregulation group alone (Fig. 5A-D). In addition, the PI3K inhibitor increased 

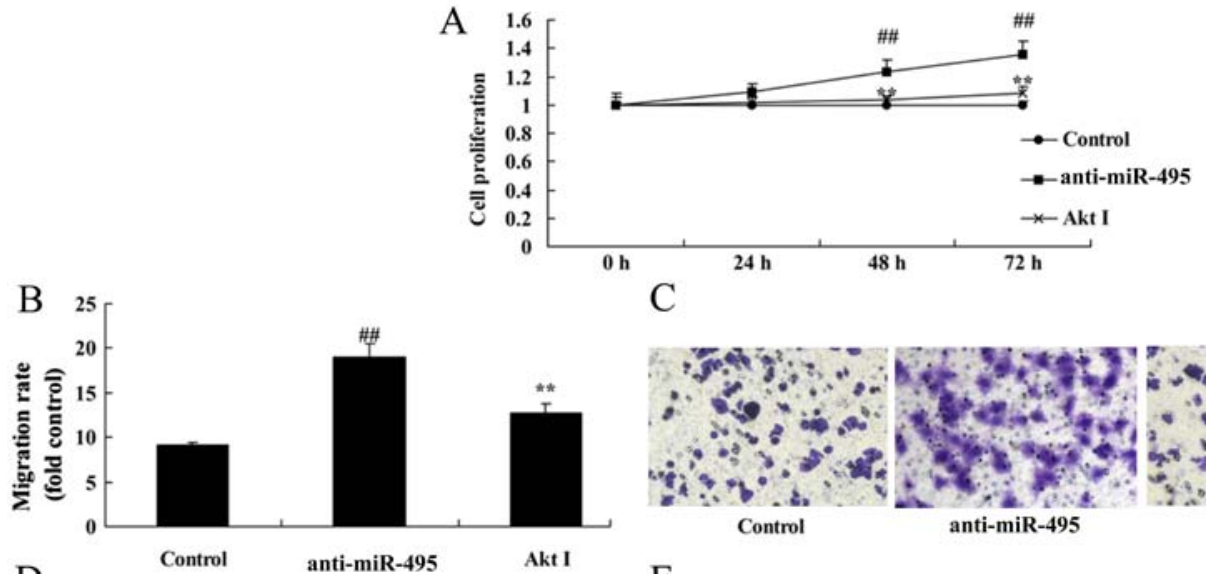

C
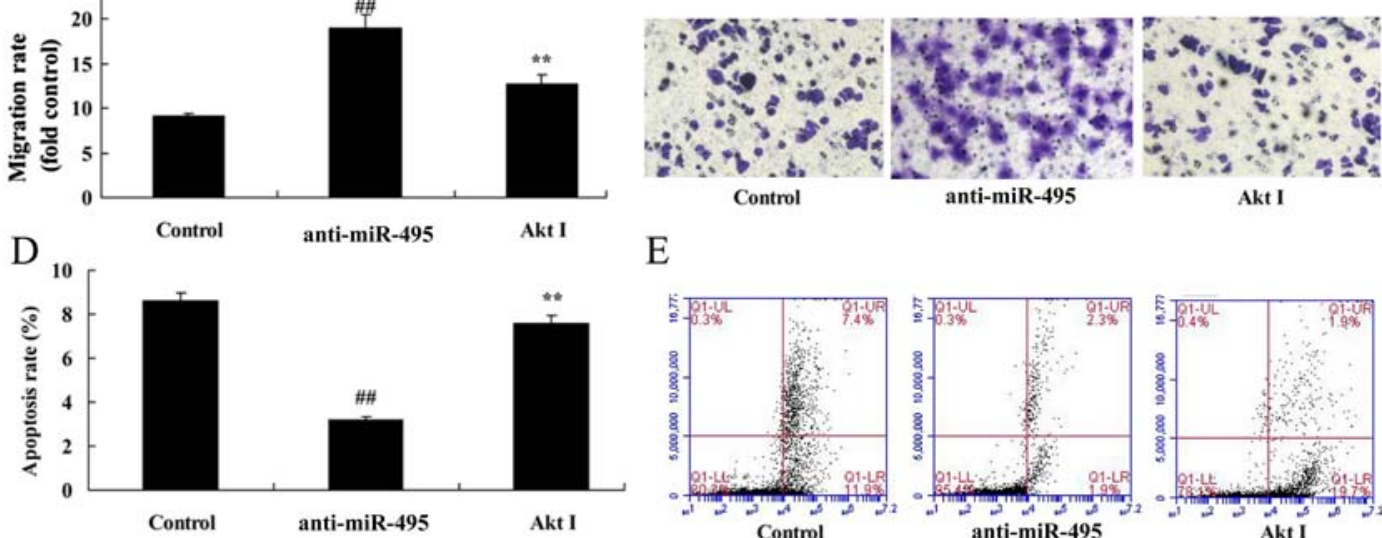

E
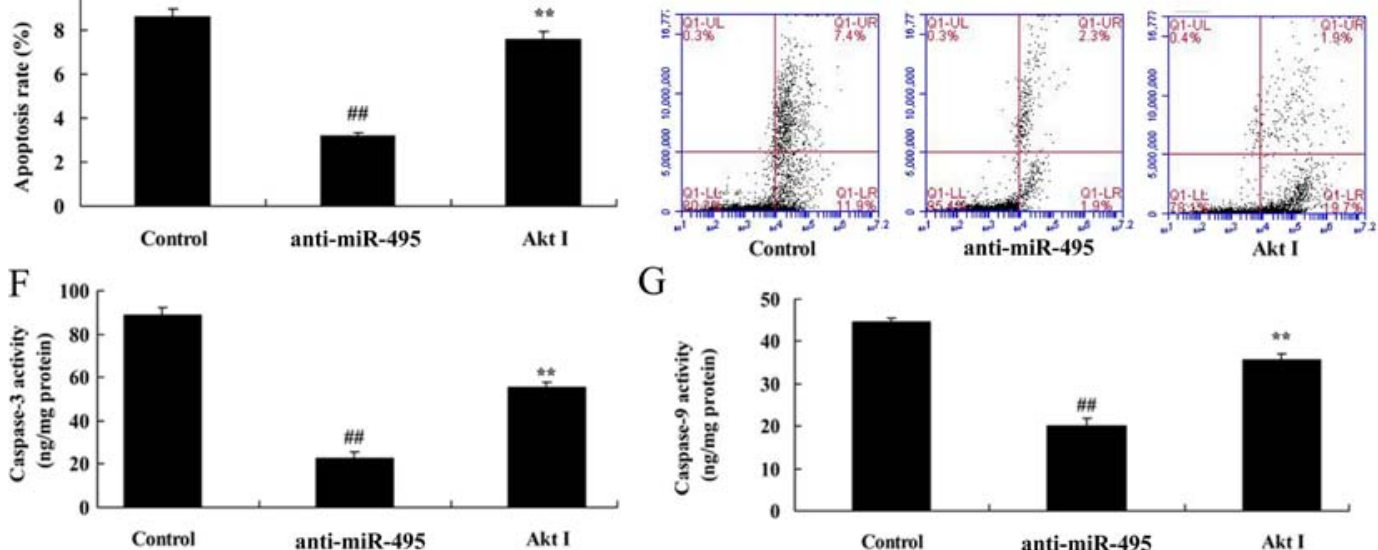

$\mathrm{G}$

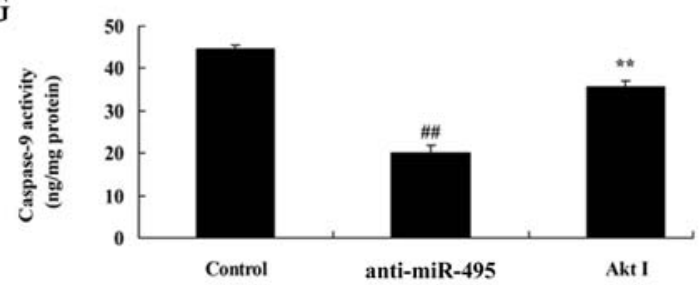

Figure 6. PI3K inhibitor inhibits cell proliferation and promotes apoptosis of gastric cancer cells following miR-495 downregulation. (A) Cell proliferation, (B and C) migration, (D and E) cell apoptosis and (F and G) caspase-3 and -9 activity, respectively. ${ }^{\# \#} \mathrm{P}<0.01$ vs. the control group, ${ }^{* *} \mathrm{P}<0.01$ vs. the downregulated miR-495 group. Control, control negative group; anti-miR-495, downregulation of the microRNA-495 group; PI3K I, downregulation of microRNA-495 and the PI3K inhibitor group
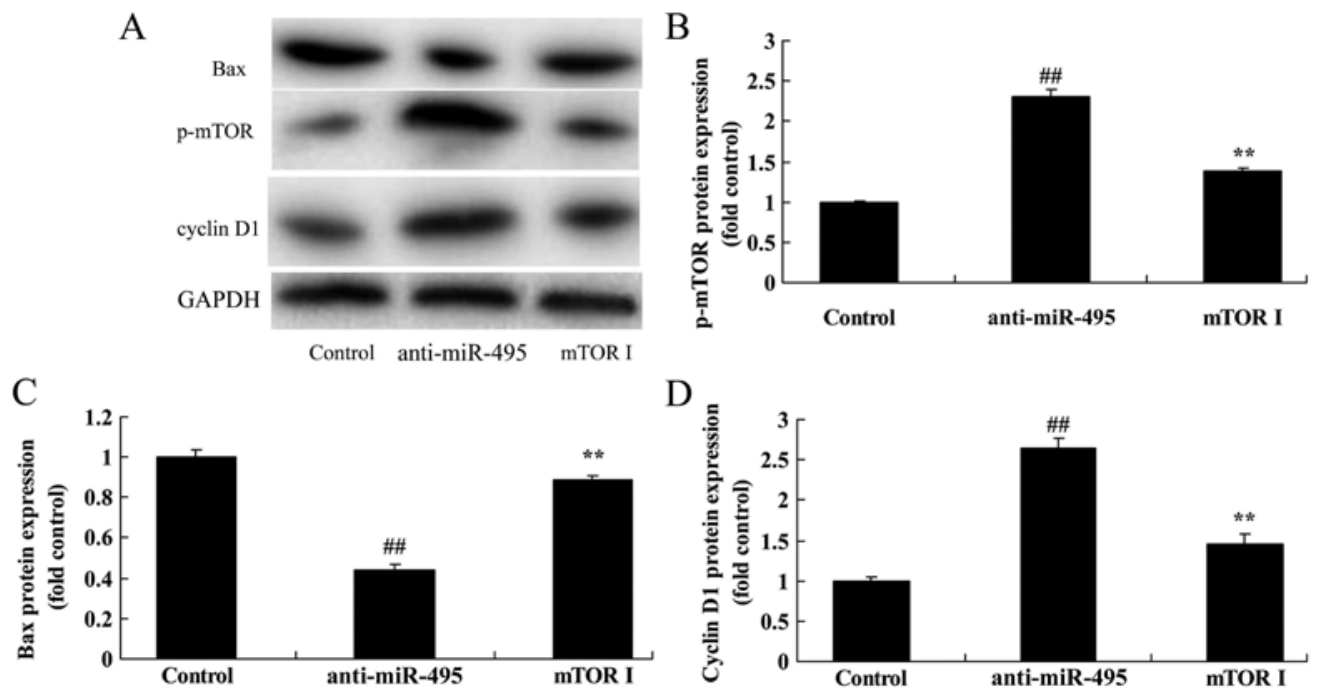

Figure 7. mTOR inhibitor suppresses the mTOR pathway of gastric cancer cells following miR-495 downregulation. (A) p-mTOR, Bax and cyclin D1 protein expression using western blot analysis and (B, C and D) p-mTOR, Bax and cyclin D1 protein expression using statistical analysis. ${ }^{\# \#<0.01 ~ v s . ~ t h e ~ c o n t r o l ~}$ group, ${ }^{* * *} \mathrm{P}<0.01$ vs. the downregulated miR-495 group. Control, control negative group; anti-miR-495, downregulation of the microRNA-495 group; mTOR I, downregulation of the microRNA-495 and the mTOR inhibitor group

Bax protein expression, and suppressed cyclin D1 protein expression in MGC80-3 cells following microRNA-495 down- regulation, compared with the miR-495 downregulation group alone (Fig. 5E-G). 

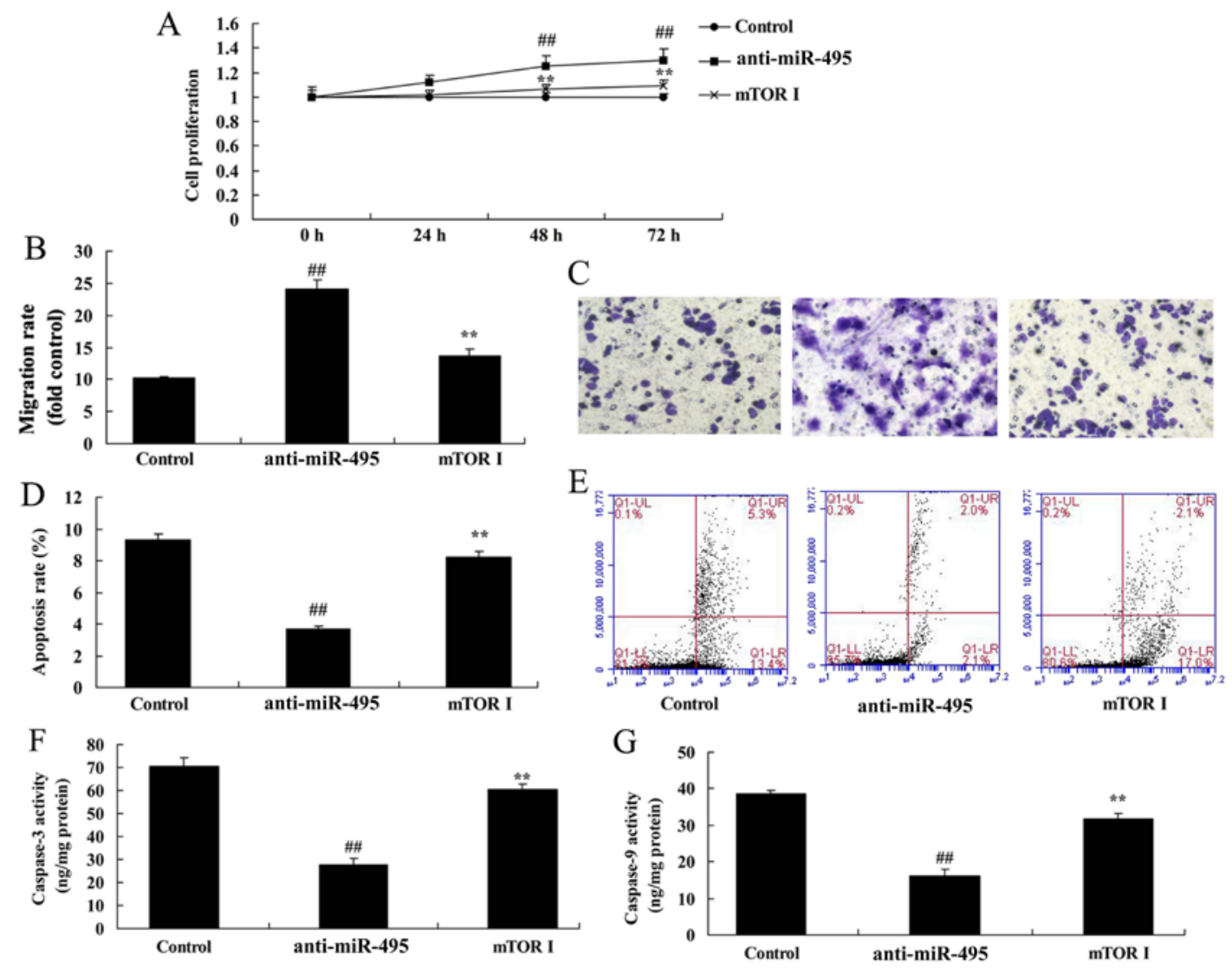

Figure 8. mTOR inhibitor inhibits cell proliferation and promotes apoptosis of gastric cancer cells following miR-495 downregulation. (A) Cell proliferation, (B and C) migration, (D and E) cell apoptosis and (F and G) caspase-3 and caspase- 9 activity. ${ }^{\# \#} \mathrm{P}<0.01$ vs. the control group, ${ }^{* *} \mathrm{P}<0.01$ vs. the downregulated miR-495 group. Control, control negative group; anti-miR-495, downregulation of the microRNA-495 group; mTOR I, downregulation of the microRNA-495 and the mTOR inhibitor group.

PI3K inhibitor inhibits cell proliferation of gastric cancer cells following miR-495 downregulation. The effects of PI3K on cell proliferation and apoptosis in gastric cancer cells in which miR-495 was downregulated, were assessed. The results revealed, a marked decrease of cell proliferation and migration, and an increase in cell apoptosis and caspase-3/-9 activity in MGC80-3 cells treated with the PI3K inhibitor following miR-495 downregulation (Fig. 6).

mTOR inhibitor suppresses the MTOR pathway of gastric cancer cells following miR-495 downregulation. Based on the previous mTOR pathway results, we assessed the effect of the inhibition of mTOR on the cell death of gastric cancer cells following miR-495 downregulation. Undoubtely, we found that the mTOR inhibitor suppressed p-mTOR and cyclin D1 protein expression, while it induced Bax protein expression of MGC80-3 cells following miR-495 downregulation, compared with the miR-495 downregulation group alone (Fig. 7).

mTOR inhibitor inhibits the cell proliferation of gastric cancer cells following miR-495 downregulation. To analyze if the mTOR axis is the function of miR-495 in gastric cancer cell apoptosis, we examined caspase-3/-9 protein expression. Our findings confirmed that the mTOR inhibitor attenuated the effect of miR-495 downregulation in the induction of cell proliferation and migration, and inhibition of apoptosis and caspase-3/-9 activity in MGC80-3 cells (Fig. 8).

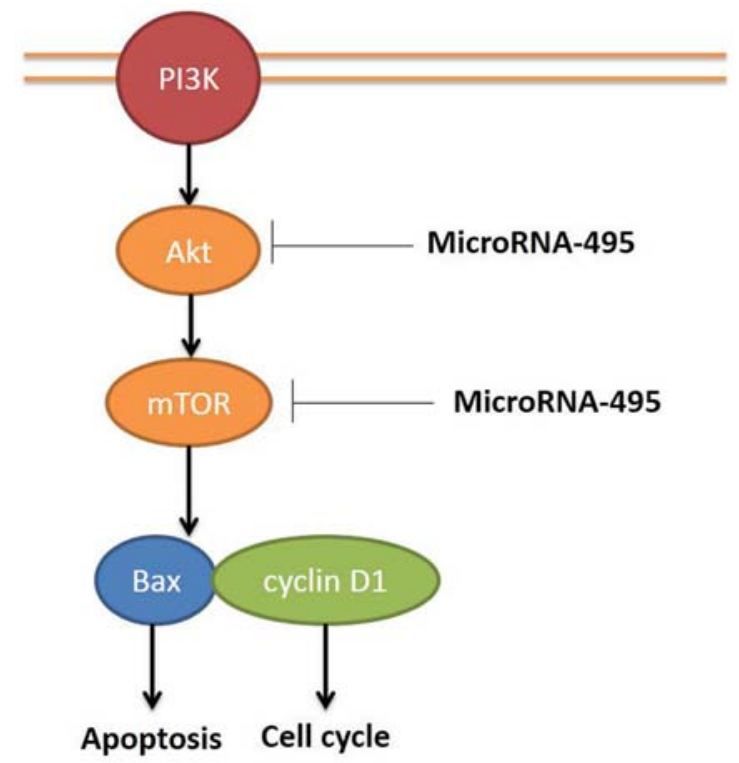

Figure 9. miR-495 regulates human gastric cancer cell apoptosis and migration through Akt and mTOR signaling. miR-495, microRNA-495.

\section{Discussion}

Gastric cancer is one of the most common malignancies in China, and its mortality is only second to lung cancer and 
liver cancer (1). A vast majority of clinically diagnosed cases are in the intermediate and advanced stage due to the lack of early specific symptoms and tumor markers for early detection and diagnosis, which has led to the high mortality of gastric cancer (13). Therefore, searching for tumor markers for the early screening or diagnosis of gastric cancer, together with developing agents with high efficiency and low toxicity is of great importance (14). The discovery of miRNAs is a milestone in the field of molecular biology (3). For the first time, we revealed that the expression level of miR-495 in patients with gastric cancer was decreased. In addition, a high expression of miR-495 increased the survival rate of gastric cancer patients. Furthermore, our data revealed that miR-495 regulated gastric cancer therapeutics. Eun et al revealed that miRNA-495-3p suppressed gastric carcinogenesis cell growth (15).

Multiple antitumor drugs exert their function through the induction of cell apoptosis and autophagy, and cell apoptosis can be achieved through death receptor-mediated cell apoptosis (16). The Bcl-2 protein family is comprised of an anti-apoptotic protein family and a pro-apoptotic protein family, which play an extremely important role in regulating cell apoptosis (17). The $\mathrm{Bcl}-2$ protein in the $\mathrm{Bcl}-2$ protein family is likely to form the Bcl-2/Bax heterodimer with pro-apoptotic protein Bax, and inhibit the pro-apoptotic effect of Bax (18). Bax can form a homodimer by itself and prompt cyto $c$ to be released in the cytoplasm, while these released pro-apoptotic factors will further induce cleavage activation of the downstream caspases, thus resulting in cell apoptosis (19). We determined that overexpression of miR-495 significantly promoted Bax protein expression, and suppressed cyclin D1 protein expression in gastric cancer cells.

Caspase-9 is one of the key proteins in the mitochondrial apoptosis pathway (20). Activated Akt can inhibit the release of mitochondrial cytochrome $c$ by changing the activities of Bcl-2 protein family members, and inhibits the activation of caspase-9. Furthermore, it can inactivate caspase-9 (Ser196) directly through phosphorylation, and suppress its pro-apoptotic effects (21). In the present study, we demonstrated that overexpression of miR-495 significantly promoted caspase-3/-9 protein expression of gastric cancer cells.

It has been indicated in recent research that PI $3 \mathrm{~K}$ is a type of intracellular phosphatidylinositol kinase that possesses the activity of serine/threonine protein kinases, and the PI3k/Akt pathway is an important anti-apoptotic pathway (8). The PI3K-mediated signal transduction pathway can regulate cell division, differentiation and apoptosis through different approaches (22). Protein kinase Akt is an important signaling molecule in the PI3K signal transduction pathway. PI3K can activate p-Akt which possesses phosphokinase activity (23). $\mathrm{p}$-Akt can increase the transcriptional activity of $\mathrm{NF}-\kappa \mathrm{B}$, upregulating the expression level of anti-apoptotic protein $\mathrm{Bcl}-2$, and promoting proliferation and invasion of tumor cells (24). Bcl-2, which is located on the mitochondrial membrane, is the anti-apoptotic protein in the downstream of the PI3K/Akt pathway (25). p-Akt can promote the binding of Bad with 14-3-3 through the promotion of the phosphorylation of Bad (Ser136), and inhibition of the pro-apoptotic effects of $\mathrm{Bad}$ (24). In addition, as the downstream of the $\mathrm{PI}$ KK/Akt pathway, mTOR activity is the key to the formation and maturation of autophagosomes (22). It is widely believed at present that mTOR is the convergence of upstream signal transduction pathways that regulate cell growth, proliferation, movement, survival and autophagy (26). The findings in our study suggest that overexpression of miR-495 suppresses the $\mathrm{PI} 3 \mathrm{~K} / \mathrm{Akt} / \mathrm{mTOR}$ pathway in gastric cancer cells. Li et al revealed that miR-495 regulated migration and invasion through Akt and mTOR signaling in prostate cancer cells (12). Results of those studies revealed that the inactivation of the $\mathrm{PI} 3 \mathrm{~K} / \mathrm{Akt} / \mathrm{mTOR}$ pathway plays a critical role in the function of miR-495-induced apoptosis of gastric cancer cells.

Inhibition of Akt can promote cell autophagy through inhibition of mTOR activity. Conversely, Akt inhibition can promote Bax expression through activation of $\mathrm{p} 53$, and enhance the activation of the mitochondrial apoptosis pathway by altering the activities of Bcl-2 pro-apoptotic protein as well as Bcl-2 anti-apoptotic protein, thus leading to cell apoptosis (24). In the present study, we demonstrated that the PI3K inhibitor, used to suppress the PI3K/Akt/mTOR pathway, inhibited cell proliferation, promoted cell apoptosis, promoted caspase-3/-9 and Bax protein expression, and suppressed cyclin D1 protein expression in gastric cancer cells through miR-495 inhibition.

In conclusion, our results indicated that miR-495 regulated human gastric cancer cell apoptosis and migration through the PI3K/Akt/mTOR pathway (Fig. 9). This finding clearly challenges the role of cell apoptosis and migration relative to the function of miR-495 for the future development of gastric cancer therapeutics.

\section{Acknowledgements}

Not applicable.

\section{Funding}

This study was supported by the Shanghai Municipal Commission of Health Natural Science Foundation (no. 20134303).

\section{Availability of data and materials}

The datasets used during the present study are available from the corresponding author upon reasonable request.

\section{Authors' contributions}

FL and FG conceived and designed the study. JW, WF, YD, $\mathrm{XM}$ performed the experiments. JW and WF wrote the paper. FL, FG, YD and XM reviewed and edited the manuscript. All authors read and approved the manuscript and agree to be accountable for all aspects of the research in ensuring that the accuracy or integrity of any part of the work are appropriately investigated and resolved.

\section{Ethics approval and consent to participate}

Samples from a previous study of patients with gastric cancer were included in the present study. Ethics approval of this previous study was obtained from the Ethics committee of Huashan Hospital, Fudan University (Shanghai, China). 


\section{Patient consent for publication}

Not applicable.

\section{Competing interests}

The authors declare that they have no competing interests

\section{References}

1. Liao W, Fu Z, Zou Y, Wen D, Ma H, Zhou F, Chen Y, Zhang M and Zhang W: MicroRNA-140-5p attenuated oxidative stress in Cisplatin induced acute kidney injury by activating Nrf2/ARE pathway through a Keap1-independent mechanism. Exp Cell Res 360: 292-302, 2017.

2. Sandborn WJ, van Assche G, Reinisch W, Colombel JF, D'Haens G, Wolf DC, Kron M, Tighe MB, Lazar A and Thakkar RB: Adalimumab induces and maintains clinical remission in patients with moderate-to-severe ulcerative colitis. Gastroenterology 142: 257-265, 2012.

3. Hua F, Ribbing J, Reinisch W, Cataldi F and Martin S: A pharmacokinetic comparison of anrukinzumab, an anti- IL-13 monoclonal antibody, among healthy volunteers, asthma and ulcerative colitis patients. Br J Clin Pharmacol 80: 101-109, 2015

4. Oliva S, Di Nardo G, Ferrari F, Mallardo S, Rossi P, Patrizi G, Cucchiara S and Stronati L: Randomised clinical trial: The effectiveness of Lactobacillus reuteri ATCC 55730 rectal enema in children with active distal ulcerative colitis. Aliment Pharmacol Ther 35: 327-334, 2012.

5. American Society for Reproductive: Revised American Society for Reproductive Medicine classification of endometriosis: 1996. Fertil Steril 67: 817-821, 1997.

6. Yamamoto Y, Hosoda K, Imahori T, Tanaka J, Matsuo K, Nakai T, Irino Y, Shinohara M, Sato N, Sasayama T, et al: Pentose phosphate pathway activation via HSP27 phosphorylation by ATM kinase: A putative endogenous antioxidant defense mechanism during cerebral ischemia-reperfusion. Brain Res 1687: 82-94, 2018.

7. Xia N, Chen G, Liu M, Ye X, Pan Y, Ge J, Mao Y, Wang H, Wang $J$ and Xie S: Anti-inflammatory effects of luteolin on experimental autoimmune thyroiditis in mice. Exp Ther Med 12: 4049-4054, 2016.

8. Eissa N,Hussein H, Kermarrec L,ElgazzarO, Metz-Boutigue MH, Bernstein CN and Ghia JE: Chromofungin (CHR: CHGA47-66) is downregulated in persons with active ulcerative colitis and suppresses pro-inflammatory macrophage function through the inhibition of NF- $\mathrm{kB}$ signaling. Biochem Pharmacol 145: 102-113, 2017.

9. Kim GD: Myricetin inhibits angiogenesis by inducing apoptosis and suppressing PI3K/Akt/mTOR signaling in endothelial cells J Cancer Prev 22: 219-227, 2017.

10. Rocha GR, Florez Salamanca EJ, de Barros AL, Lobo CIV and Klein MI: Effect of tt-farnesol and myricetin on in vitro biofilm formed by Streptococcus mutans and Candida albicans. BMC Complement Altern Med 18: 61, 2018.

11. Cho BO, Yin HH, Park SH, Byun EB, Ha HY and Jang SI: Anti-inflammatory activity of myricetin from Diospyros lotus through suppression of NF- $\kappa \mathrm{B}$ and STAT1 activation and Nrf2-mediated HO-1 induction in lipopolysaccharide-stimulated RAW264.7 macrophages. Biosci Biotechnol Biochem 80: 1520-1530, 2016.
12. Li JZ, Wang ZL, Xu WH, Li Q, Gao L and Wang ZM: MicroRNA-495 regulates migration and invasion in prostate cancer cells via targeting Akt and mTOR signaling. Cancer Invest 34: 181-188, 2016.

13. Zhao L, Qi Y, Xu L, Tao X, Han X, Yin L and Peng J: MicroRNA-140-5p aggravates doxorubicin-induced cardiotoxicity by promoting myocardial oxidative stress via targeting Nrf2 and Sirt2. Redox Biol 15: 284-296, 2018.

14. Obora K, Onodera Y, Takehara T, Frampton J, Hasei J, Ozaki T, Teramura $\mathrm{T}$ and Fukuda K: Inflammation-induced miRNA-155 inhibits self-renewal of neural stem cells via suppression of CCAAT/enhancer binding protein $\beta(\mathrm{C} / \mathrm{EBP} \beta)$ expression. Sci Rep 7: 43604, 2017.

15. Eun JW, Kim HS, Shen Q, Yang HD, Kim SY, Yoon JH, Park WS, Lee JY and Nam SW: MicroRNA-495-3p functions as a tumour suppressor by regulating multiple epigenetic modifiers in gastric carcinogenesis. J Pathol 244: 107-119, 2018.

16. Scott FI and Lichtenstein GR: Biosimilars in the treatment of inflammatory bowel disease: Supporting evidence in 2017. Curr Treat Options Gastroenterol 16: 147-164, 2018.

17. LeeHand Lee CS:Flavonoid myricetininhibits TNF- $\alpha$-stimulated production of inflammatory mediators by suppressing the Akt, mTOR and NF- $\kappa \mathrm{B}$ pathways in human keratinocytes. Eur J Pharmacol 784: 164-172, 2016.

18. Rosario M, French JL, Dirks NL, Sankoh S, Parikh A, Yang H, Danese S, Colombel JF, Smyth M, Sandborn WJ, et al: Exposure-efficacy relationships for vedolizumab induction therapy in patients with ulcerative colitis or Crohn's disease. J Crohn's Colitis 11: 921-929, 2017.

19. Xie J and Zheng Y: Myricetin protects keratinocyte damage induced by UV through I $\mathrm{B} / \mathrm{NF}$ kb signaling pathway. J Cosmet Dermatol 16: 444-449, 2017.

20. Allamneni C, Venkata K, Yun H, Xie F, DeLoach L and Malik TA: comparative effectiveness of vedolizumab vs. infliximab induction therapy in ulcerative colitis: Experience of a real-world cohort at a tertiary inflammatory bowel disease center. Gastroenterol Res 11: 41-45, 2018.

21. Robbins L, Zaghiyan K, Melmed G, Vasiliauskas E, Ahmed S, McGovern D, Rabizadeh S, Singh N, Landers C, Ippoliti A, et al: Outcomes with anti-tumour necrosis factor-alpha therapy and serology in patients with denovo Crohn's disease after ileal pouch anal anastomosis. J Crohn's Colitis 11: 77-83, 2017.

22. Yuan CX, Zhou ZW, Yang YX, He ZX, Zhang X, Wang D, Yang T, Pan SY, Chen XW and Zhou SF: Danusertib, a potent pan-Aurora kinase and ABL kinase inhibitor, induces cell cycle arrest and programmed cell death and inhibits epithelial to mesenchymal transition involving the PI3K/Akt/mTOR-mediated signaling pathway in human gastric cancer AGS and NCI-N78 cells. Drug Des Devel Ther 9: 1293-1318, 2015.

23. Gu P, Zhu L, Liu Y, Zhang L, Liu J and Shen H: Protective effects of paeoniflorin on TNBS-induced ulcerative colitis through inhibiting NF-kappaB pathway and apoptosis in mice. Int Immunopharmacol 50: 152-160, 2017.

24. Tavares M, de Lima C, Fernandes W, Martinelli V, de Lucena M, Lima F, Telles A, Brandão L and de Melo Júnior M: Tumour necrosis factor-alpha (-308G/A) promoter polymorphism is associated with ulcerative colitis in Brazilian patients. Int J Immunogenet 43: 376-382, 2016.

25. Yu ZH, Huang F, Xu N, Zhao DM, Hu FA, Liu J and Liu HF: Expression of Toll-like receptor 4, CD14, and NF- $\mathrm{BB}$ in Chinese patients with ulcerative colitis. J Immunoassay Immunochem 32: 47-56, 2011.

26. Gálvez-Llompart M, Recio MC and García-Domenech R: Topological virtual screening: A way to find new compounds active in ulcerative colitis by inhibiting NF- $\kappa$ B. Mol Divers 15: 917-926, 2011. 